\title{
Self-Similarity, Fractality and Entropy Principle in Collisions of Hadrons and Nuclei at Tevatron, RHIC and LHC
}

\section{Zborovský}

The Czech Academy of Science, Nuclear Physics Institute, Řež, Czech Republic

E-mail: zborovskyaujf.cas.cz

\section{Tokarev}

Joint Institute for Nuclear Research, Dubna, Russia

E-mail: tokarevejinr.ru

\begin{abstract}
$z$-Scaling of inclusive spectra as a manifestation of self-similarity and fractality of hadron interactions is illustrated. The scaling for negative particle production in $A u+A u$ collisions from BES-I program at RHIC is demonstrated. The scaling variable $z$ depends on the momentum fractions of the colliding objects carried by the interacting constituents, and momentum fractions of the produced objects in the scattered and recoil directions, which carries the inclusive particle and its counterpart, respectively. Structures of the colliding objects and fragmentation processes in final state are expressed by fractal dimensions. Medium produced in the collisions is described by a specific heat. The scaling function $\psi(z)$ reveals energy, angular, multiplicity, and flavor independence. It has a power behavior at high $z\left(\right.$ high $\left.p_{T}\right)$. Based on entropy principle and $z$-scaling, energy loss as a function of the collision energy, centrality and transverse momentum of inclusive particle is estimated. New conservation law including fractal dimensions is found. Quantization of fractal dimensions is discussed.
\end{abstract}

40th International Conference on High Energy physics - ICHEP2020

July 28 - August 6, 2020

Prague, Czech Republic (virtual meeting)

\footnotetext{
* Speaker.
} 


\section{1. $z$-Scaling}

One of the behavior related to the self-similarity and fractality of hadron interactions is manifested by the $z$-scaling of inclusive particle production [1]-[4]. The scaling variable $z=z_{0} \cdot \Omega^{-1}$ is proportional to the quantity $z_{0}=\sqrt{s_{\perp}} /\left[\left(d N_{c h} /\left.d \eta\right|_{0}\right)^{c} m_{N}\right]$, where $\sqrt{s_{\perp}}$ is the transverse kinetic energy of a selected binary sub-process responsible for production of the inclusive particle with mass $m_{a}$ and its partner (antiparticle) with mass $m_{b}$. The multiplicity density $d N_{c h} /\left.d \eta\right|_{0}$ of charged particles in the central interaction region, the nucleon mass $m_{N}$, and the parameter $c$, interpreted as a "specific heat" of the produced medium, completely determine the value of $z_{0}$. The quantity $\Omega$ is the maximal relative number of parton configurations containing binary sub-processes defined by the momentum fractions $x_{1}$ and $x_{2}$ of colliding hadrons (nuclei), and by the momentum fractions $y_{a}$ and $y_{b}$ of objects created directly in these sub-processes, which carry the inclusive particle and its counterpart, respectively. The relative number of the configurations is given by the function $\Omega=\left(1-x_{1}\right)^{\delta_{1}}\left(1-x_{2}\right)^{\delta_{2}}\left(1-y_{a}\right)^{\varepsilon_{a}}\left(1-y_{b}\right)^{\varepsilon_{b}}$, where $\delta_{1}$ and $\delta_{2}$ are fractal dimensions of the colliding objects, and $\varepsilon_{a}$ and $\varepsilon_{b}$ are fractal dimensions of the fragmentation processes in the scattered and recoil direction, respectively. The selected binary sub-process is defined by the maximum of $\Omega\left(x_{1}, x_{2}, y_{a}, y_{b}\right)$ with the kinematic constraint $\left(x_{1} P_{1}+x_{2} P_{2}-p / y_{a}\right)^{2}=\left(x_{1} M_{1}+x_{2} M_{2}+m_{b} / y_{b}\right)^{2}$, where $P_{1}, P_{2}$ and $M_{1}, M_{2}$ are 4-momenta and masses of the colliding objects, $p$ is 4-momentum of the inclusive particle and $m_{b}$ is mass of its counterpart, respectively. The function $\Omega^{-1}$ represents a resolution at which a sub-process defined by the fractions $x_{1}, x_{2}, y_{a}, y_{b}$ can be singled out of the inclusive reaction. The scaling variable $z$ has property of a fractal measure. It grows in a power-like manner with the increasing resolution $\Omega^{-1}$. The scaling function [2]

$$
\psi(z)=\frac{\pi}{(d N / d \eta) \sigma_{i n}} J^{-1} E \frac{d^{3} \sigma}{d p^{3}}
$$

is expressed in terms of the measurable quantities and Jacobian $\left\{p_{T}^{2}, y\right\} /\{z, \eta\}$. The scale transformation $z \rightarrow \alpha_{F} z, \quad \psi \rightarrow \alpha_{F}^{-1} \psi$ is used for comparison of $\psi(z)$ for different hadron species $(F)$.

\section{Self-similarity of hadron production in $p+p$ collisions}

The main properties of $z$-presentation of the inclusive differential cross sections of particles produced in proton-(anti)proton collisions are the energy, angular, and multiplicity independence of the scaling function $\psi(z)$ for different types of hadrons, direct photons and jets, confirmed by numerous data obtained at U70, ISR, Sp $\overline{\mathrm{p}} \mathrm{S}$, Tevatron, RHIC and LHC. Figure 1(a) shows $z$ presentation [1] of the spectra of $\pi^{-}, K^{-}, \bar{p}$, and $\Lambda^{\prime} s$ produced in $p+p$ collisions at the energies $\sqrt{s}=19-200 \mathrm{GeV}$ and $\theta_{c m s}=3^{0}-90^{\circ}$. The $z$-scaling was obtained for $\delta_{1}=\delta_{2} \equiv \delta$ and $\varepsilon_{a}=$ $\varepsilon_{b} \equiv \varepsilon_{F}$. The energy and angular independence of $\psi(z)$ was found for $c=0.25, \delta=0.5$ and for the stated values of $\varepsilon_{F}$ and $\alpha_{F}$. Figure 1(b) shows $p_{T}$-dependence of $y_{a}$ for $\pi^{-}, K^{-}$mesons and antiprotons at the energy $\sqrt{s}=19,53,200 \mathrm{GeV}$ and $\theta_{c m s}=90^{\circ}$. All curves demonstrate a growth with $p_{T}$. It means that the relative energy dissipation $\Delta E_{q} / E_{q}=\left(1-y_{a}\right)$ associated with the production of a high- $p_{T}$ particle is smaller than for the inclusive process at lower transverse momenta. The decrease of $y_{a}$ with the increasing $\sqrt{s}$ corresponds to more energy dissipation at higher energies. The slight decrease of $y_{a}$ with the mass of the inclusive particle means greater energy dissipation in the production of heavier hadrons compared to lighter ones. 


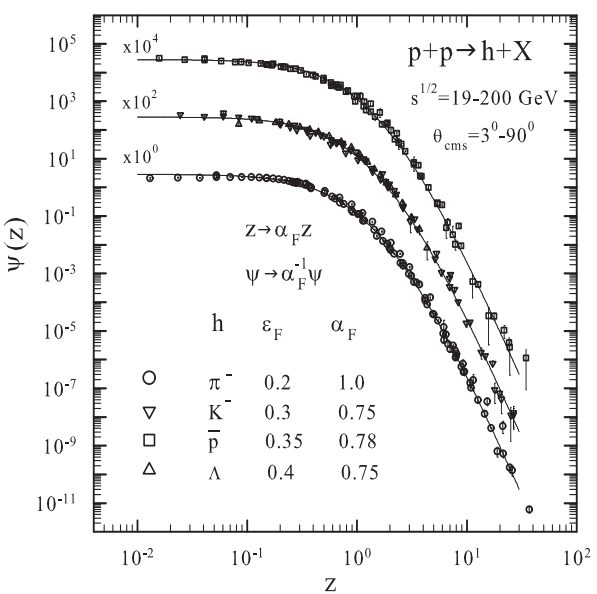

(a)

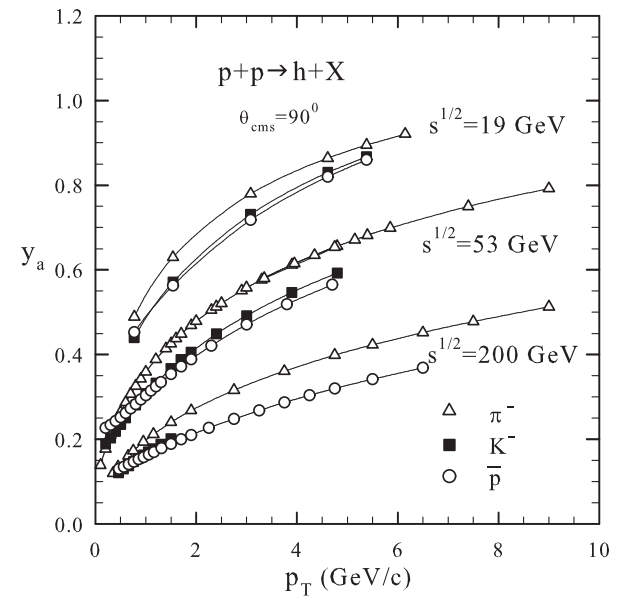

(b)

Figure 1: (a) The inclusive spectra of $\pi^{-}, K^{-}, \bar{p}$, and $\Lambda$ hadrons produced in $p+p$ collisions in $z-$ presentation [1]. (b) The $p_{T}$ dependence of the momentum fraction $y_{a}$ for $\pi^{-}, K^{-}$, and $\bar{p}$ produced in $p+p$ collisions at $\sqrt{s}=19,53$, and $200 \mathrm{GeV}$ in the central rapidity region.

The flavor independence of $\psi(z)$ for $K_{S}^{0}, K^{-}, K^{* 0}, \phi$ mesons and $\Lambda, \Lambda^{*}, \Sigma^{*}, \Xi, \Omega$ hyperons measured by the STAR and PHENIX Collaborations in $p+p$ collisions was studied in [2]. The $z$-scaling of the strange hadrons was confirmed for the model parameters which correspond to larger energy losses than for pions. The energy loss was found to be larger for strange baryons than for strange mesons. The growth indicates increasing tendency with larger number of strange valence quarks inside the strange baryon, $(\Delta E / E)_{\Omega}>(\Delta E / E)_{\Xi^{-}}>(\Delta E / E)_{\Lambda} \simeq(\Delta E / E)_{\Lambda^{*}} \simeq(\Delta E / E)_{\Sigma^{*}}$.

The flavor independence of $\psi(z)$ was tested also for the top quark [4]. The $z$-presentation of the spectra of top quark obtained in $p+p$ collisions at the LHC energies $\sqrt{s}=7,8$, and $13 \mathrm{TeV}$ was compared with data on the top-quark production obtained by the D $\varnothing$ Collaboration at $\sqrt{s}=$ $1.96 \mathrm{TeV}$. The same values of $\delta=0.5$ and $c=0.25$ were used as for other hadrons. We have set $\varepsilon_{t o p}=0$, as negligible energy loss is assumed in the elementary $t \bar{t}$ production process. It was shown that the LHC and Tevatron data on inclusive spectra of top quark support the flavor independence of $\psi(z)$ over the interval of $z=0.01-8$ for $\alpha_{t o p} \simeq 0.0045$.

We analyzed [4] data on inclusive cross sections of jet production in $p+p$ collisions at the LHC energies $\sqrt{s}=2760,7000$, and $8000 \mathrm{GeV}$ in the framework of $z$-scaling. The results were compared with $z$-presentation of jet spectra at the energies $\sqrt{s}=630,1800,1960 \mathrm{GeV}$. The data obtained at the LHC confirm results of the analysis [5] of jet spectra measured by the DØ and CDF Collaborations in $\bar{p}+p$ collisions with parameters $c=1, \delta=1$, and $\varepsilon_{j e t}=0$. The scaling function can be described by a power law $\psi(z) \sim z^{-\beta}$ over a wide range of $z=60-20000$.

\section{Self-similarity of negative hadron production in $A u+A u$ collisions}

We extended the applicability of the self-similarity principle to the description of hadron production in nucleus-nucleus collisions. The self-similarity concerns fractal structure of the colliding objects, interaction of their constituents and fractal character of fragmentation processes in the final state. This physical principle is assumed to be valid also in the high-density and hightemperature phase in which quark and gluon degrees of freedom dominate. Figure 2(a) shows the 


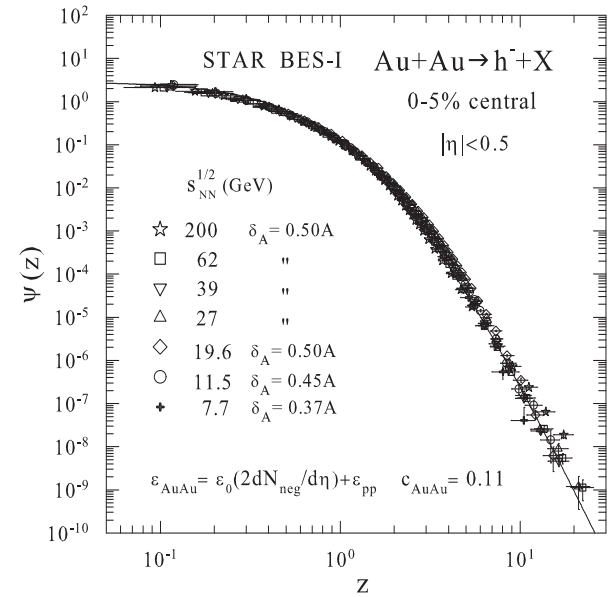

(a)

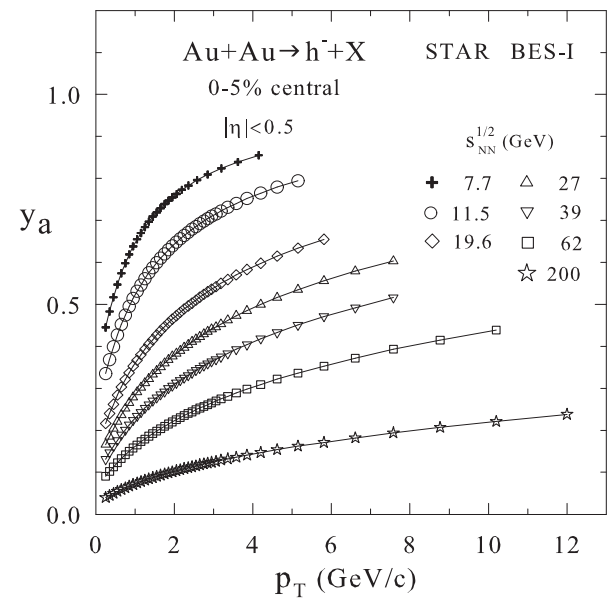

(b)

Figure 2: (a) The scaling function $\psi(z)$ and (b) the momentum fraction $y_{a}$ in dependence on $p_{T}$ for negative hadrons produced in $(0-5) \%$ central $A u+A u$ collisions at $\sqrt{s_{N N}}=7.7,11.5,19.6,27,39,62.4$ and $200 \mathrm{GeV}$ [3]. The symbols correspond to experimental data [6] measured by the STAR Collaboration at RHIC.

function $\psi(z)$ for negative hadrons [3] produced in $(0-5) \%$ central $A u+A u$ collisions at different $\sqrt{s_{N N}}=7.7-200 \mathrm{GeV}$. The symbols correspond to spectra [6] measured by the STAR Collaboration at the pseudorapidity $|\eta|<0.5$. The $z$-presentation of the spectra demonstrates energy independence of $\psi(z)$ over the analyzed kinematic range. The symbols nearly coincide with the solid curve depicting $z$-scaling of $h^{-}$particles produced in $p+p$ collisions. The same energy independence of $\psi(z)$ is valid [3] for different centrality classes of $A u+A u$ collisions.

The scaling was obtained for $\varepsilon_{A u A u}=\varepsilon_{0}\left(2 d N_{n e g}^{A u A u} / d \eta\right)+\varepsilon_{p p}$ with a suitable choice of $\varepsilon_{0}$ and for the constant values of the model parameters $c_{A u A u}=0.11, \delta_{A}=A \delta, \delta=0.5$, and $\varepsilon_{p p}=0.2$ at $\sqrt{s_{N N}} \gtrsim 19.6 \mathrm{GeV}$. The parameter $\varepsilon_{0}$ shows [3] a logarithmic increase with $\sqrt{s_{N N}}$. It reflects the growing suppression of hadron yields in the central collisions of heavy nuclei. The increase of $\varepsilon_{A u A u}$ with produced multiplicity $N_{c h}^{A A} \simeq 2 N_{n e g}^{A A}$ is connected with a decrease of $y_{a}$ representing larger energy loss at higher energies and centralities. Figure 2(b) shows the dependence of the fraction $y_{a}$ on $p_{T}$ for $h^{-}$hadrons produced in $(0-5) \%$ central $A u+A u$ collisions for different energies. A growth of $y_{a}$ with $p_{T}$ and its decrease with $\sqrt{s_{N N}}$ correspond to the diminishing of relative energy loss at high transverse momentum and its increase at higher collision energies.

\section{4. $z$-Scaling and maximum entropy principle}

The parameters used in the $z$-scaling scheme can be interpreted in terms of thermodynamic quantities (entropy, specific heat, etc.) [1]. The scaling variable is proportional to the ratio of the energy $\sqrt{s_{\perp}}$ and the maximal value of $W\left(x_{1}, x_{2}, y_{a}, y_{b}\right)=\left(d N_{c h} /\left.d \eta\right|_{0}\right)^{c} \cdot \Omega\left(x_{1}, x_{2}, y_{a}, y_{b}\right)$, constrained by a kinematic condition. The function $W$ is proportional to the number of all parton and hadron configurations of the colliding system which contain the constituent configuration defined by particular values of the momentum fractions $x_{1}, x_{2}, y_{a}$ and $y_{b}$. The absolute number of the configurations, $W_{S}=W \cdot W_{0}$, is given up to a constant $W_{0}$. We write the entropy of the configurations 
as follows

$$
S=c \cdot \ln \left(d N_{c h} /\left.d \eta\right|_{0}\right)+\ln \left(1-x_{1}\right)^{\delta_{1}}\left(1-x_{2}\right)^{\delta_{2}}\left(1-y_{a}\right)^{\varepsilon_{a}}\left(1-y_{b}\right)^{\varepsilon_{b}}+\ln W_{0}
$$

The multiplicity density characterizes a "temperature" and $c$ "specific heat" of the produced matter. The second term in (4.1) depends on the volume in the space of the momentum fractions.

\subsection{Conservation of fractal cumulativity}

There exists a scale dependent quantity characterizing hadron interactions at a constituent level which is conserved. The conservation law reflects a symmetry of transformation of one fractal structure into another one at all scales. The statement is based on maximization of the entropy (4.1) with a kinematic constraint. It gives specific dependences of the momentum fractions on the kinematics of the inclusive reaction. The momentum fractions satisfy the equality [7]

$$
\delta_{1} \frac{x_{1}}{1-x_{1}}+\delta_{2} \frac{x_{2}}{1-x_{2}}=\varepsilon_{a} \frac{y_{a}}{1-y_{a}}+\varepsilon_{b} \frac{y_{b}}{1-y_{b}} .
$$

This equation represents a conservation law for the quantity $C(D, \zeta)=D \cdot g(\zeta)$, where $D$ means fractal dimension, $g(\zeta)=\zeta /(1-\zeta)$, and $\zeta$ is the corresponding momentum fraction. The conservation law holds for any inclusive reaction with arbitrary momenta $P_{1}, P_{2}$ and $p$ of the colliding and inclusive particles. We name the quantity $C(D, \zeta)$ as the "fractal cumulativity" of a fractal-like structure with the dimension $D$ carried by its constituent with the momentum fraction $\zeta$. The conservation law is formulated as follows: The fractal cumulativity before a constituent interaction is equal to that after the constituent interaction for any binary sub-process,

$$
\sum_{i}^{\text {in }} C\left(D_{i}, \zeta_{i}\right)=\sum_{j}^{\text {out }} C\left(D_{j}, \zeta_{j}\right)
$$

The quantity $C(D, \zeta)$ characterizes property of a fractal-like object or a fractal-like process with the dimension $D$ to form a structural aggregate with certain degree of local compactness, which carries its momentum fraction $\zeta$.

\subsection{Quantization of fractal dimensions}

The fractal character of hadron structure and fragmentation processes manifests itself most prominently near the kinematic limit $\left(x_{1}, x_{2}, y_{a}, y_{b}\right) \rightarrow 1$. In this region, the momentum dependent part of the entropy (4.1) can be written [7] in the form $S_{\Upsilon}-S_{\Gamma}$, where $S_{\Upsilon}$ depends on momenta and masses, and $S_{\Gamma}=\left(\delta_{1}+\delta_{2}+\varepsilon_{1}+\varepsilon_{2}\right) \ln \left(\delta_{1}+\delta_{2}+\varepsilon_{1}+\varepsilon_{2}\right)-\delta_{1} \ln \delta_{1}-\delta_{2} \ln \delta_{2}-\varepsilon_{a} \ln \varepsilon_{a}-\varepsilon_{b} \ln \varepsilon_{b}$ depends solely on fractal dimensions. It allows us to derive physical consequences provided the fractal dimensions are expressed as integer multiples $\delta_{1}=n_{\delta_{1}} \cdot d, \delta_{2}=n_{\delta_{2}} \cdot d, \varepsilon_{a}=n_{\varepsilon_{a}} \cdot d, \varepsilon_{b}=n_{\varepsilon_{b}} \cdot d$ of the same constant $d$. Based on the quantization of fractal dimensions, the entropy $S_{\Gamma}$ can be interpreted within a statistical ensemble of the interacting fractal configurations as follows. The statistical ensemble is considered as a large collection of $n_{\delta_{1}}$ fractals with random configurations but with the same fractal dimension $\delta_{1}$, together with an analogous set of $n_{\delta_{2}}$ interacting fractals with the fractal dimension $\delta_{2}$, which are combined via binary sub-processes with the collection of $n_{\varepsilon_{a}}$ 
fractals with random configurations but with the same fractal dimension $\varepsilon_{a}$, and the corresponding set of $n_{\varepsilon_{b}}$ fractals with the fractal dimension $\varepsilon_{b}$. The entropy $S_{\Gamma}$ can be rewritten into the form

$$
S_{\Gamma}=d \cdot \ln \frac{\left(n_{\delta_{1}}+n_{\delta_{2}}+n_{\varepsilon_{a}}+n_{\varepsilon_{b}}\right) !}{n_{\delta_{1}} ! \cdot n_{\delta_{2}} ! \cdot n_{\varepsilon_{a}} ! \cdot n_{\varepsilon_{b}} !} .
$$

According to statistical physics, this gives us possibility to interpret the entropy $S_{\Gamma}$, expressed in units of the dimensional quantum $d$, as the logarithm of the number of different ways, in which the fractal dimensions of the interacting fractal structures can be composed from the identical dimensional quanta, each of the size $d$.

The quantization of fractal dimensions, $D=n_{D} \cdot d$, is connected with quantum character of the fractal cumulativity. This can be written as $C(D, \zeta)=n_{C} \cdot d$, where $n_{C}\left(n_{D}, \zeta\right)=n_{D} \cdot \zeta /(1-\zeta)$ represents the number of quanta of the fractal cumulativity expressed in units of the dimensional quantum $d$. The quantum character of the fractal dimensions has profound impact on the physical content of the conservation law for fractal cumulativity. According to (4.2), the number of cumulativity quanta is conserved at any resolution given by the momenta $P_{1}, P_{2}$ and $p$ of the colliding and inclusive particles. The conservation law can be formulated as follows: The number of quanta of fractal cumulativity before a constituent interaction is equal to that after the constituent interaction for any binary sub-process,

$$
\sum_{i}^{\text {in }} n_{C}\left(n_{D_{i}}, \zeta_{i}\right)=\sum_{j}^{\text {out }} n_{C}\left(n_{D_{j}}, \zeta_{j}\right)
$$

The quantization of the dimension $D$ and the cumulativity $C(D, \zeta)$ is based on the assumptions of the fractal self-similarity of internal hadron structure, fractal nature of fragmentation process, and locality of hadron interactions at a constituent level up to the kinematic limit.

\section{Conclusions}

The $z$-scaling is a specific feature of particle production reflecting the self-similarity, locality, and fractality of hadron interactions at a constituent level. The analysis of the STAR BES-I data indicates validity of the $z$-scaling for negative hadrons produced in $A u+A u$ system. Using the principle of maximum entropy, the variable $z$ reflects the conservation of the fractal cumulativity, quantization of the fractal dimensions, and conservation of the number of cumulativity quanta [8].

\section{References}

[1] I. Zborovský, M. V. Tokarev, Phys. Rev. D 75, 094008 (2007); Int. J. Mod. Phys. A 24, 1417 (2009).

[2] M. V. Tokarev, I. Zborovský, Int. J. Mod. Phys. A 32, 1750029 (2017).

[3] M. Tokarev, A. Kechechyan, I. Zborovský, Nucl. Phys. A 993, 121646 (2020).

[4] M. V. Tokarev, I. Zborovský, A. O. Kechechyan, T. G. Dedovich, Phys. Part. Nucl. 51, 141 (2020).

[5] M. V. Tokarev, I. Zborovský, T. G. Dedovich, Int. J. Mod. Phys. A 27, 1250115 (2012).

[6] M. Tokarev (for the STAR Collab.), Int. J. Mod. Phys. Conf. Ser. 39, 1560103 (2015).

[7] I. Zborovský, Int. J. Mod. Phys. A 33, 1850057 (2018).

[8] I. Zborovský, M. Tokarev, JINR, Dubna Report No. E2-2020-24, 2020; arXiv:2010.08330. 